\title{
Primary endpoint analysis of the multicentre phase II hypo-FLAME trial for intermediate and high risk prostate cancer
}

Cédric Draulans ${ }^{1,2}$, Uulke A. van der Heide ${ }^{3}$, Karin Haustermans ${ }^{1,2}$, Floris J. Pos ${ }^{3}$, Jochem van der Voort van Zyp ${ }^{4}$, Hans De Boer ${ }^{4}$, Veerle H. Groen ${ }^{4}$, Evelyn M. Monninkhof ${ }^{4,5}$, Robert J. Smeenk ${ }^{6}$, Martina KunzeBusch $^{6}$, Robin De Roover ${ }^{1,2}$, Tom Depuydt ${ }^{1,2}$, Sofie Isebaert ${ }^{1,2}$, Linda G.W. Kerkmeijer ${ }^{4,6}$

\begin{tabular}{|c|c|}
\hline Cédric Draulans ${ }^{1,2}$, M.D. & cedric.draulans@uzleuven.be \\
\hline Uulke A. van der Heide ${ }^{3}$, PhD & u.vd.heide@nki.nl \\
\hline Karin Haustermans ${ }^{1,2}$, M.D., PhD & karin.haustermans@uzleuven.be \\
\hline Floris Pos ${ }^{3}$, M.D. & f.pos@nki.nl \\
\hline Jochem van der Voort van Zyp ${ }^{4}$, M.D., PhD & J.R.N.vanderVoortvanZyp@umcutrecht.nl \\
\hline Hans De Boer ${ }^{4}, \mathrm{PhD}$ & J.C.J.deBoer-6@umcutrecht.nl \\
\hline Veerle H. Groen ${ }^{4}$, M.D. & V.H.Groen@umcutrecht.nl \\
\hline Evelyn M. Monninkhof ${ }^{4,5}, \mathrm{PhD}$ & E.Monninkhof@umcutrecht.nl \\
\hline Robert J. Smeenk ${ }^{6}$, M.D., PhD & RobertJan.Smeenk@radboudumc.nl \\
\hline Martina Kunze-Busch ${ }^{6}, \mathrm{PhD}$ & Martina.Kunze-Busch@radboudumc.nl \\
\hline Robin De Roover ${ }^{1,2}$ & robin.deroover@uzleuven.be \\
\hline Tom Depuydt ${ }^{1,2}$, PhD & tom.depuydt@uzleuven.be \\
\hline Sofie Isebaert ${ }^{1,2}, \mathrm{PhD}$ & sofie.isebaert@uzleuven.be \\
\hline Linda G.W. Kerkmeijer ${ }^{4,6}$, M.D., PhD & Linda.Kerkmeijer@radboudumc.nl \\
\hline
\end{tabular}

\footnotetext{
${ }^{1}$ Department of Radiation Oncology, University Hospitals Leuven, Leuven, Belgium.

2 Department of Oncology, KU Leuven, Leuven, Belgium.

${ }^{3}$ Department of Radiation Oncology, The Netherlands Cancer Institute, Amsterdam, The Netherlands.

${ }^{4}$ Department of Radiation Oncology, University Medical Center, Utrecht, The Netherlands.

5 Julius Centre for Health Sciences and Primary Care, University Medical Centre Utrecht, Utrecht, The Netherlands

${ }^{6}$ Department of Radiation Oncology, Radboud University Medical Centre, Nijmegen, The Netherlands.
}

\section{Conflicts of interest: None}

\section{$\underline{\text { Keywords }}$}

Prostatic neoplasms; Radiotherapy; Stereotactic body radiotherapy; Focal boost; Extreme hypofractionation 


\begin{abstract}
Background and purpose: Local recurrences after radiotherapy for prostate cancer (PCa) often originate at the location of the macroscopic tumour(s). Since PCa cells are known to be sensitive to high fraction doses, hypofractionated whole gland stereotactic body radiotherapy (SBRT) in conjunction with a simultaneous ablative microboost to the macroscopic tumour(s) within the prostate could be a way to reduce the risk of local failure. We investigated the safety of this treatment strategy.
\end{abstract}

Materials and methods: Patients with intermediate or high risk PCa were enrolled in a prospective phase II trial, called hypo-FLAME. All patients were treated with extreme hypofractionated doses of 35 Gy in 5 weekly fractions to the whole prostate gland with an integrated boost up to 50 Gy to the multiparametric (mp) MRI-defined tumour(s). Treatment related toxicity was measured using the CTCAE v4.0. The primary endpoint of the trial was treatment related acute toxicity.

Results: Between April 2016 and December 2018, 100 men were treated in 4 academic centres. All patients were followed up for a minimum of 6 months. The median mean dose delivered to the visible tumour nodule(s) on mpMRI was $44.7 \mathrm{~Gy}$ in this trial. No grade $\geq 3$ acute genitourinary (GU) or gastrointestinal (GI) toxicity was observed. Furthermore, 90 days after start of treatment, the cumulative acute grade $2 \mathrm{GU}$ and $\mathrm{GI}$ toxicity rates were $34.0 \%$ and $5.0 \%$, respectively.

Conclusion: Simultaneous focal boosting to the macroscopic tumour(s) in addition to whole gland prostate SBRT is associated with acceptable acute GU and GI toxicity. 


\section{Introduction}

Prostate cancer ( $\mathrm{PCa}$ ) is the second most common cancer and the fifth leading cause of cancer death in men worldwide [1]. External beam radiotherapy (EBRT) is an appropriate treatment for a large proportion of patients with intermediate or high risk PCa. Dose escalation studies with conformal EBRT, targeting the whole prostate gland, showed improved biochemical disease-free survival (bDFS), distant metastases-free survival and even overall survival [2-5]. However, when escalating the dose to the whole gland, these positive results come at the expense of increased toxicity $[6,7]$. Since many patients have prolonged survival after radiation treatment for $\mathrm{PCa}$, dose escalation should only be pursued in the context of acceptable toxicity.

Traditionally, standard EBRT for PCa has been delivered in fractions of 1.8 to 2.0 Gy spread across 7 to 9 weeks. Evidence from preclinical studies and clinical trials suggest an enhanced sensitivity to higher doses per fraction for prostate tumours, reflected by a low $\alpha / \beta$ ratio [8]. A significant implication thereof is that hypofractionation may allow to biologically escalate the dose while maintaining current levels of toxicity $[9,10]$. Three noninferiority randomized clinical trials on moderate hypofractionation, using fractions of 2.5 to $3.0 \mathrm{~Gy}$, demonstrated the efficacy and safety of this approach [11-13].

Delivering even higher fraction doses, i.e. $\geq 5 \mathrm{~Gy} /$ fraction, is possible by means of stereotactic body radiotherapy (SBRT), also named ultra-hypofractionation or extreme hypofractionation. SBRT is a specific subcategory of EBRT employing sophisticated radiotherapy techniques at high accuracy which allow the delivery of such large radiation doses per fraction [14]. Furthermore, better clinical outcome results for high risk PCa patients are also expected to be achieved by dose escalation in hypofractionated schedules, in congruence with the results of dose escalation in conventional EBRT trials [2]. Recently, the HYPO-RT-PC phase III trial reported non-inferior results regarding both failurefree survival and long term toxicity for a seven-fraction ultra-hypofractionated radiotherapy schedule compared to conventionally fractionated radiotherapy for intermediate-to-high risk PCa [15]. Besides, one phase III trial (PACE-B) and one pooled data analysis of multiple cohort studies showed, respectively, acceptable toxicity rates and good biochemical control rates for low and intermediate risk PCa patients when 33.5 to 40.0 Gy was delivered in 4 to 5 fractions [16,17]. Furthermore, stepwise dose escalation up to $50 \mathrm{~Gy}$ in 5 fractions to the whole prostate gland proved to result into excellent bDFS. This treatment regimen, however, appeared to be associated with notably more gastrointestinal (GI) and genitourinary (GU) toxicity $[18,19]$.

Since it has been shown that local recurrences occur most often at the location of the macroscopic tumour(s) prior to treatment [20], an alternative strategy to perform dose escalation is to deliver a simultaneously integrated boost to the intraprostatic tumour(s). Given that, in this strategy, the dose 
is being escalated only to the macroscopic intraprostatic tumour nodule(s) instead of the whole prostate gland, the toxicity profile might be more favourable. This treatment technique was previously demonstrated to be safe and feasible for conventional EBRT by the multicentre randomised controlled phase III FLAME trial [21,22]. Today, multiparametric magnetic resonance imaging (mpMRI) including T2-weighted (T2w), diffusion-weighted (DWI) and dynamic contrast enhanced (DCE) imaging is most often used for intraprostatic tumour delineation. Van Houdt et al. showed that DCE and DWI images provide complementary information reflecting the PCa heterogeneity [23].

By using hypofractionated whole gland SBRT in conjunction with focal tumour boosting, one could combine the potential advantages of both strategies. Following previous phase I trials on focal boosting by SBRT [24-27], we conducted a phase II trial, called the hypo-FLAME-trial, investigating the safety of delivering an ablative microboost to the macroscopic tumour(s) within the prostate using extreme hypofractionation. In the present paper, we report the trial's primary endpoint on treatment related acute side effects.

\section{Materials and methods}

This multicentre prospective phase II hypo-FLAME study (NCT02853110, ClinicalTrials.gov) was carried out in three centres in the Netherlands, i.e. University Medical Centre Utrecht (UMCU) (Utrecht), Netherlands Cancer Institute/Antoni van Leeuwenhoek Hospital (NKI-AvL) (Amsterdam), and Radboud University Medical Center (RadboudUMC) (Nijmegen), and in one centre in Belgium, i.e. University Hospitals Leuven (UZL) (Leuven). This study was approved by the institutional ethical review boards of UMCU for the Netherlands (NL53719.041.15a) and UZL for Belgium (s59632). Informed written consent was obtained from all patients participating in the trial.

\section{Patient selection}

Inclusion criteria were men over 18 years of age with histologically confirmed diagnosis of adenocarcinoma of the prostate. Only patients with intermediate or high risk PCa with at least one of the following risk features were eligible: clinical T-stage T2b, T2c, T3a or T3b on mpMRI (TNM $7^{\text {th }}$ edition), Gleason score $\geq 7$ ( $\geq$ ISUP grade 2 ) or iPSA $\geq 10 \mathrm{ng} / \mathrm{mL}$. If seminal vesicle invasion was $\geq 5 \mathrm{~mm}$ diagnosed on mpMRI or iPSA was $>30 \mathrm{ng} / \mathrm{mL}$, patients were excluded. In addition, at least one tumour nodule needed to be visible on mpMRI for trial inclusion. Patients were excluded if they had prior pelvic radiotherapy or a prior transurethral resection of the prostate (TURP), evidence of lymph node or distant metastases, severe lower urinary tract symptoms (International Prostate Symptom Score (IPSS) $\geq 15$ ) or a low performance status (World Health Organization (WHO) score $>2$ ). Finally, if gold fiducial 
marker insertion was unsafe or if there was any contraindication to perform a mpMRI, patients were also considered ineligible.

\section{Radiotherapy planning and delivery}

All included patients were scheduled to receive $35 \mathrm{~Gy}$ in 5 weekly fractions to the whole prostate gland over 29 days. This corresponds with a 2 Gy equivalent dose (EQD2) of 85 Gy using the linear quadratic formula and assuming an $\alpha / \beta$ ratio of $1.5 \mathrm{~Gy}$ for PCa. Simultaneously, an additional integrated iso-toxic focal boost up to $50 \mathrm{~Gy}$ was planned to the macroscopic tumour nodule(s) visualized on mpMRI.

For delineation of organs at risk (OARs) and target volumes, computed tomography (CT) and mpMRI examinations were performed. The mpMRI examination consisted of T2w, DWI and DCE imaging according to the Prostate Imaging - Reporting and Data System Version 2 (PI-RADS ${ }^{\text {TM }}$ V2) in treatment position [28]. The planning CT was co-registered with the mpMRI scan in treatment position. The planning $\mathrm{CT}$ and all treatments were performed with a comfortably filled bladder. At one out of the four participating centres (RadboudUMC) a rectal balloon was used. At the three other centres, in case of an extremely full rectum on cone beam CT (CBCT), patients were advised to empty their bowel or to use enemas. All institutes performed transperineal or transrectal implantation of three or four gold seed fiducial markers (median: 4, range 3-4) for precise target positioning.

Visible tumour nodule(s) on mpMRI were contoured as gross tumour volume (GTV) in collaboration with an experienced uroradiologist. The whole prostate gland, including a $4 \mathrm{~mm}$ margin around the GTV for microscopic extracapsular extension and excluding OARs, was considered clinical target volume (CTV). The seminal vesicles were contoured up to the discretion of the treating physician according to the ESTRO ACROP consensus guideline on CT- and MRI-based target volume delineation for primary radiation therapy of localised PCa [29]. The margin from CTV to planned target volume (PTV) was $4(n=63)$ or $5(n=37) \mathrm{mm}$ based on earlier experience with image-guided volumetric modulated arc therapy (VMAT) prostate SBRT [30]. VMAT was performed in all participating institutes. The rectum, anal canal, bladder, small bowel, femoral heads, penile bulb and urethra were delineated as OARs [31]. Afterwards a $2 \mathrm{~mm}$ isotropic planning risk volume (PRV) margin was used for both the rectum and urethra to limit high dose exposure. The prescription dose to the prostate PTV was 33.25 $\mathrm{Gy}(95 \%$ of $35 \mathrm{~Gy}$ ), and the prescription dose to the GTV was $35 \mathrm{~Gy}$ with an iso-toxic boost up to $50 \mathrm{~Gy}$. The focal boost dose was escalated as high as achievable while maintaining the OAR dose constraints. No limit on target dose heterogeneity was specified by the protocol, but effort was made to limit the maximum dose $\left(D_{0.1 c c}\right)$ to $\leq 52 \mathrm{~Gy}$. The detailed planning objectives are summarized in Supplemental Table S1. 
Treatment planning software was used to create dual-arc VMAT plans with photon energies $>6 \mathrm{MV}$. Patients were treated on C-arm linear accelerators. Daily on-line position verification of the prostate was performed either by orthogonal on-board kV x-ray imaging or cone-beam CT [32]. The choice for combining EBRT with androgen deprivation therapy (ADT), as well as the aimed duration of this ADT treatment was at the discretion of the treating radiation oncologist with no specific treatment intervention required by the study protocol.

\section{Primary endpoint}

The primary outcome measure was acute GI and GU toxicity measured using the Common Terminology Criteria for Adverse Events version 4.0 (CTCAE v4.0). Acute toxicity is defined as toxicity occurring within 90 days after the first radiation treatment [33]. GI and GU toxicities were recorded at baseline, weekly during treatment, at 90 days after the first radiation treatment and at 6 months after the last treatment fraction. The following adverse events were scored as part of GI toxicity scoring: abdominal pain, diarrhea, enterocolitis, fecal incontinence, flatulence, haemorrhoids, proctitis, rectal fistula, rectal haemorrhage and rectal pain. As part of GU toxicity, noninfective cystitis, hematuria, urinary frequency, urinary incontinence, urinary retention and urinary tract pain were scored. The safety outcome in the SBRT setting was considered as primary endpoint, since the efficacy of focal boosting is simultaneously investigated in the phase III FLAME study [21].

\section{Sample size and statistical analysis}

The trial was powered to detect $a \geq 6 \%$ increase of the acute toxicity incidence grade $\geq 3$ compared to the acute toxicity percentages reported during and after conventional EBRT for PCa by Lips et al. [34]. To achieve a power of $82 \%$ with a one-sided significance level of 0.05 , the inclusion of at least 100 patients was needed. Patient characteristics are summarized as median in combination with range for continuous variables and as proportions for categorical variables. Toxicity scores up to 6 months after treatment are presented as well as the prevalence and cumulative prevalence of GU and GI toxicity. Furthermore, target and OAR dose parameters are reported descriptively and interquartile ranges (IQR) were calculated for each parameter. Physical doses (SBRT) were converted into EQD2, using the linear quadratic formula and assuming an $\alpha / \beta$ ratio of $1.5 \mathrm{~Gy}$ for $\mathrm{PCa}$ and an $\alpha / \beta$ ratio of $3 \mathrm{~Gy}$ for OARs. Data were analysed using SPSS 25 for Windows (IBM Corp., Armonk, NY, USA).

\section{Results}

Between April 2016 and December 2018, 100 patients were enrolled and treated at 4 hospital centres. Baseline characteristics of all enrolled patients are shown in Table 1. The median age at the start of 
the radiation treatment of all included patients was 73 years (range, 57-84 years) and the median follow-up time was 18 months (range, 6-30 months). Furthermore, 75\% of the patients were classified as high risk PCa patients, while the other $25 \%$ were classified as intermediate risk PCa patients according to the EAU risk classification [35]. The median initial PSA level was $10.8 \mathrm{ng} / \mathrm{mL}$ (range, 3.0$29.0 \mathrm{ng} / \mathrm{mL}$ ) and the largest group of patients (44\%) was staged clinical tumour category cT3a, which implies the suspicion of extracapsular tumour extension. Based on pretreatment biopsy findings $57 \%$ of the patients had a Gleason Score of 7. If Grade 4 was mentioned as the predominant pattern, they were classified ISUP grade 3 (24\%), otherwise ISUP grade 2 (33\%). Sixty-two percent of the included patients were intended to receive ADT of which $31 \%$ for short term ( $\leq 6$ months) and $31 \%$ for long term (6-36 months). The median overall treatment time was 29 days (range, 27-36 days).

The median mean dose ( $D_{\text {mean }}$ ) delivered to the GTV, defined as the visible tumour nodule(s) on mpMRI, was $44.7 \mathrm{~Gy}$ (37.7 Gy - 50.9 Gy), which correlates with a converted EQD2 of $133.3 \mathrm{~Gy}$ when an $\alpha / \beta$ ratio of $1.5 \mathrm{~Gy}$ is applied for PCa. Furthermore, the median dose received by $99 \%$ of the GTV volume (Dو9) was $40.3 \mathrm{~Gy}$ (36.2 Gy - $50.7 \mathrm{~Gy}$ ). The median volume of the GTV per patient was $2.3 \mathrm{cc}$. The median dose to $1 \mathrm{cc}\left(\mathrm{D}_{1 \mathrm{cc}}\right)$ of the rectum and bladder were $35.0 \mathrm{~Gy}$ (31.4 Gy - 36.5 Gy) and 36.1 (34.4 Gy $-40.3 \mathrm{~Gy}) \mathrm{Gy}$, respectively. The median maximum point dose ( $\left.\mathrm{D}_{0.035 \mathrm{cc}}\right)$ to the urethra was $39.3 \mathrm{~Gy}$ (36.5 Gy - 41.4 Gy). Detailed dose statistics for each structure are summarized in Table 2.

The 90 -days cumulative incidence of grade $2 \mathrm{GU}$ toxicity was $34.0 \%$. No grade $\geq 3$ acute GU toxicity was observed. Furthermore, 90 days after start of treatment, the cumulative acute grade $2 \mathrm{GI}$ toxicity rates were $5.0 \%$. Similar to results on GU toxicity, no grade $\geq 3$ acute GI toxicity was observed. The prevalence of grade $\geq 1, \geq 2$ and $\geq 3 \mathrm{Gl}$ and $\mathrm{GU}$ events over time until 6 months after radiotherapy is shown in Figure 1. When observing the toxicity scores as a function of time, the prevalence of $\mathrm{GU}$ toxicity reached a maximum at week 5 , with $25.5 \%$ of the patients suffering from grade 2 toxicity. A decline in GU grade 2 toxicity to $11.4 \%$ is observed, 90 days after starting the radiation treatment. The prevalence of $\mathrm{GI}$ grade 2 toxicity did not exceed $5 \%$ at any timepoint. The distribution pattern of the acute scored CTCAE v4.0 items is shown in Table 3.

\section{Discussion}

In this phase II hypo-FLAME trial, we found that delivering a focal ablative microboost up to $50 \mathrm{~Gy}$ to the macroscopic tumour nodule(s) within the prostate using extreme hypofractionation is safe in terms of acute toxicity for patients with intermediate and high risk PCa. Notably, the proportion of patients 
free from acute grade $\geq 3$ toxicity were considerably lower $(0 \%)$ than the $3 \%$ grade 3 acute toxicity reported for conventional radiotherapy by Lips et al. [34].

When compared with other recent extreme hypofractionation trials, the GU and GI toxicity rates reported in the hypo-FLAME trial fall within a similar range. The PACE-B phase III trial randomized low and intermediate risk PCa patients to on the one hand conventional or moderate hypofractionated radiotherapy (78 Gy in 39 fractions over 7.5 weeks, or 62 Gy in 20 fractions over 4 weeks) and on the other hand extreme hypofractionated radiotherapy ( $36.25 \mathrm{~Gy}$ in 5 fractions over 1 to 2 weeks weeks) $[16,36]$. Analysis of acute toxicity in this PACE-B trial showed 23.2\% Radiation Therapy Oncology Group (RTOG) scale grade $\geq 2$ acute GU toxicity and $10.2 \%$ RTOG scale grade $\geq 2$ acute GI toxicity. Furthermore, a pooled individual patient data analysis of 12 extreme hypofractionation cohort studies including 2142 patients by Kishan et al. was recently published [17]. These studies reported a crude incidence of acute GU and GI composite RTOG and CTCAE grade $\geq 3$ toxicity of $0.6 \%$ and $0.09 \%$, respectively. A direct comparison of the toxicity data of the different trials is difficult given the differences in patient selection and the known interscale (CTCAE vs RTOG) variability [37]. This being said, these comparisons demonstrate the acceptability of the reported toxicities in the hypo-FLAME study in terms of both grade and prevalence, even with the addition of a focal boost dose to the tumour. The priority of the dose constraints to the OARs above the aimed dose of the focal boost probably explains why there is no increase in toxicity despite the performed dose-escalation.

Lastly, we compared our results with the cumulative acute toxicity results from the randomized controlled phase III FLAME trial, investigating the benefit of a focal boost to the visible tumour(s) inside the prostate in a conventional radiotherapy fractionation schedule [22]. For the focal boost arm, the reported cumulative incidence for grade $\geq 2$ acute GU and $\mathrm{Gl}$ toxicity was $42.3 \%$ and $14.8 \%$, respectively. Potential hypotheses regarding the $8.3 \%$ lower reported cumulative acute GU toxicity and the 9.8\% lower reported cumulative acute GI toxicity include a biological advantage of extreme hypofractionation, sharper dose gradients that are created with SBRT and an intensified attention for motion management in the SBRT setting.

To our knowledge, this is the largest phase II trial to date evaluating the safety of escalating the radiation therapy dose to the dominant intraprostatic nodule(s) using SBRT. Earlier phase I trials suggested a dose up to $50 \mathrm{~Gy}$ to the dominant intraprostatic nodule(s) as recommended aimed dose for focal boosting [25-27]. In our trial, the median mean dose delivered to the intraprostatic tumour(s) was $44.7 \mathrm{~Gy}$ instead of the aimed $50 \mathrm{~Gy}$, due to priority of normal tissue constraints. The location of the intraprostatic tumour(s) relatively to the rectum and urethra mainly determined the boost dose level. Nevertheless, the median mean dose delivered to the intraprostatic tumour(s) was a substantial 
boost compared to the dose delivered to the whole prostate gland $(+27.7 \%)$. Furthermore, the biologically delivered median mean dose in the hypo-FLAME trial (EQD2 = 133.3 Gy) was notably higher than that delivered in the previous FLAME trial (EQD2 $=106.3 \mathrm{~Gy}$ ), using an $\alpha / \beta$ ratio of $1.5 \mathrm{~Gy}$ for PCa [22]. Further technical details about the hypo-FLAME trial and the position of the applied strategy in the SBRT spectrum were extensively discussed in [32].

The reported follow-up of the included patients is so far limited to 6 months posttreatment, and further follow-up is warranted. However, in the previously mentioned pooled individual patient data analysis by Kishan et al., reporting on both acute and late toxicity with 7 years follow-up, a multivariable logistic regression was performed to predict late $\mathrm{GU}$ and GI grade $\geq 3$ toxicity. By this, reported acute composite RTOG and CTCAE grade 3 or higher GU and GI toxicity was found as a strong predictor (odds ratio, 19.42; 95\% confidence interval, 5.14-73.42; $p=0.008$ ) for late GU and GI toxicity [17]. Based on our own results, we expect late toxicity will be acceptable in our study cohort considering the demonstrated favourable acute toxicity results. While the hypo-FLAME study is also collecting patient-reported data, only physician-reported data were considered in this first analysis on acute toxicity. Further follow-up is planned to definitively evaluate long-term toxicity, quality of life and tumour related outcome.

Currently, the potential benefit of SBRT, also in high-risk patients, is being investigated in several randomized trials. These trials include PACE-C (NCT01584258), HEAT (NCT01794403), and NRG GU005 (NCT03367702). However, if a significant benefit of focal boosting would be found by the randomised phase III FLAME trial (NCT01168479), the FLAME schedule may become the new standard of care. A subsequent randomized trial comparing SBRT with focal boost (hypo-FLAME) to the more fractionated focal boost treatment (FLAME) would be the most logical next step.

While the clinical benefit of focal dose escalation is still under investigation in the phase III FLAME trial, the current phase II hypo-FLAME trial showed that a focal SBRT boost to the macroscopic tumour(s) is associated with acceptable acute GU and GI toxicity in addition to whole gland prostate SBRT. Furthermore, besides the potential benefit in tumour control by focal boosting and extreme hypofractionation, the associated reduction in fraction number is attractive to both patients and radiation oncology departments.

\section{Registration number ClinicalTrials.gov}

NCT02853110 


\section{Conflict of interest statement}

All authors declare having no conflict of interest related to the content of this manuscript.

\section{Acknowledgements}

Research project funded by Kom op tegen Kanker (Stand up to Cancer), the Flemish cancer society.

We thank Saskia van Amelsvoort and Cees Haaring for their efforts in the hypo-FLAME trial management. 


\section{References}

[1] Bray F, Ferlay J, Soerjomataram I, Siegel RL, Torre LA, Jemal A. Global cancer statistics 2018: GLOBOCAN estimates of incidence and mortality worldwide for 36 cancers in 185 countries. CA Cancer J Clin 2018;68:394-424. doi:10.3322/caac.21492.

[2] Viani GA, Stefano EJ, Afonso SL. Higher-than-conventional radiation doses in localized prostate cancer treatment: a meta-analysis of randomized, controlled trials. Int J Radiat Oncol Biol Phys 2009;74:1405-18. doi:10.1016/j.ijrobp.2008.10.091.

[3] Kupelian PA, Ciezki J, Reddy CA, Klein EA, Mahadevan A. Effect of increasing radiation doses on local and distant failures in patients with localized prostate cancer. Int J Radiat Oncol Biol Phys 2008;71:16-22. doi:10.1016/j.ijrobp.2007.09.020.

[4] Zelefsky MJ, Pei X, Chou JF, Schechter M, Kollmeier M, Cox B, et al. Dose Escalation for Prostate Cancer Radiotherapy: Predictors of Long-Term Biochemical Tumor Control and Distant Metastases?Free Survival Outcomes. Eur Urol 2011;60:1133-9. doi:10.1016/j.eururo.2011.08.029.

[5] Pahlajani N, Ruth KJ, Buyyounouski MK, Chen DYT, Horwitz EM, Hanks GE, et al. Radiotherapy doses of $80 \mathrm{~Gy}$ and higher are associated with lower mortality in men with Gleason score 8 to 10 prostate cancer. Int J Radiat Oncol Biol Phys 2012;82:1949-56.

doi:10.1016/j.jijrobp.2011.04.005.

[6] Dearnaley DP, Jovic G, Syndikus I, Khoo V, Cowan RA, Graham JD, et al. Escalated-dose versus control-dose conformal radiotherapy for prostate cancer: long-term results from the MRC RT01 randomised controlled trial. Lancet Oncol 2014;15:464-73. doi:10.1016/S14702045(14)70040-3.

[7] Al-Mamgani A, van Putten WL, Heemsbergen WD, van Leenders GJLH, Slot A, Dielwart MFH, et al. Update of Dutch Multicenter Dose-Escalation Trial of Radiotherapy for Localized Prostate Cancer. Int J Radiat Oncol 2008;72:980-8. doi:10.1016/j.ijrobp.2008.02.073.

[8] Miralbell R, Roberts SA, Zubizarreta E, Hendry JH. Dose-fractionation sensitivity of prostate cancer deduced from radiotherapy outcomes of 5,969 patients in seven international institutional datasets: $\alpha / \beta=1.4$ (0.9-2.2) Gy. Int J Radiat Oncol Biol Phys 2012;82:e17-24. doi:10.1016/j.jjrobp.2010.10.075.

[9] Cabrera AR, Lee WR. Hypofractionation for clinically localized prostate cancer. Semin Radiat Oncol 2013;23:191-7. doi:10.1016/j.semradonc.2013.01.005.

[10] Ritter M. Rationale, Conduct, and Outcome Using Hypofractionated Radiotherapy in Prostate Cancer. Semin Radiat Oncol 2008;18:249-56.

[11] Lee WRR, Dignam JJJ, Amin MB, Bruner DW, Low D, Swanson GPP, et al. Randomized phase III noninferiority study comparing two radiotherapy fractionation schedules in patients with lowrisk prostate cancer. J Clin Oncol 2016;34:2325-32. doi:10.1016/j.ijrobp.2015.10.049.

[12] Dearnaley D, Syndikus I, Mossop H, Khoo V, Birtle A, Bloomfield D, et al. Conventional versus hypofractionated high-dose intensity-modulated radiotherapy for prostate cancer: 5-year outcomes of the randomised, non-inferiority, phase $3 \mathrm{CHHiP}$ trial. Lancet Oncol 2016;17:1047-60. doi:10.1016/S1470-2045(16)30102-4.

[13] Catton CN, Lukka H, Julian JA, Gu C-S, Martin J, Supiot S, et al. A randomized trial of a shorter radiation fractionation schedule for the treatment of localized prostate cancer. J Clin Oncol 2016;34. 
[14] Morgan SC, Hoffman K, Loblaw DA, Buyyounouski MK, Patton C, Barocas D, et al. Hypofractionated Radiation Therapy for Localized Prostate Cancer: An ASTRO, ASCO, and AUA Evidence-Based Guideline. Pract Radiat Oncol 2018;8:354-60. doi:10.1200/JCO.18.01097.

[15] Widmark A, Gunnlaugsson A, Beckman L, Thellenberg-Karlsson C, Hoyer M, Lagerlund M, et al. Ultra-hypofractionated versus conventionally fractionated radiotherapy for prostate cancer: 5 -year outcomes of the HYPO-RT-PC randomised, non-inferiority, phase 3 trial. Lancet 2019;394:385-95. doi:10.1016/S0140-6736(19)31131-6.

[16] Brand DH, Tree AC, Ostler P, van der Voet H, Loblaw A, Chu W, et al. Intensity-modulated fractionated radiotherapy versus stereotactic body radiotherapy for prostate cancer (PACE-B): acute toxicity findings from an international, randomised, open-label, phase 3 , non-inferiority trial. Lancet Oncol 2019;0. doi:10.1016/S1470-2045(19)30569-8.

[17] Kishan AU, Dang A, Katz AJ, Mantz CA, Collins SP, Aghdam N, et al. Long-term Outcomes of Stereotactic Body Radiotherapy for Low-Risk and Intermediate-Risk Prostate Cancer. JAMA Netw Open 2019;2:e188006. doi:10.1001/jamanetworkopen.2018.8006.

[18] Boike TP, Lotan Y, Cho LC, Brindle J, DeRose P, Xie X-J, et al. Phase I dose-escalation study of stereotactic body radiation therapy for low- and intermediate-risk prostate cancer. J Clin Oncol 2011;29:2020-6. doi:10.1200/JCO.2010.31.4377.

[19] Kim DWN, Cho LC, Straka C, Christie A, Lotan Y, Pistenmaa D, et al. Predictors of rectal tolerance observed in a dose-escalated phase 1-2 trial of stereotactic body radiation therapy for prostate cancer. Int J Radiat Oncol Biol Phys 2014;89:509-17. doi:10.1016/j.ijrobp.2014.03.012.

[20] Cellini N, Morganti AG, Mattiucci GC, Valentini V, Leone M, Luzi S, et al. Analysis of intraprostatic failures in patients treated with hormonal therapy and radiotherapy: implications for conformal therapy planning. Int J Radiat Oncol 2002;53:595-9. doi:10.1016/S0360-3016(02)02795-5.

[21] Lips IM, van der Heide UA, Haustermans K, van Lin EN, Pos F, Franken SP, et al. Single blind randomized Phase III trial to investigate the benefit of a focal lesion ablative microboost in prostate cancer (FLAME-trial): study protocol for a randomized controlled trial. Trials 2011;12:255. doi:10.1186/1745-6215-12-255.

[22] Monninkhof EM, van Loon JWL, van Vulpen M, Kerkmeijer LGW, Pos FJ, Haustermans K, et al. Standard whole prostate gland radiotherapy with and without lesion boost in prostate cancer: Toxicity in the FLAME randomized controlled trial. Radiother Oncol 2018;127:74-80. doi:10.1016/j.radonc.2017.12.022.

[23] van Houdt PJ, Ghobadi G, Schoots IG, Heijmink SWTPJ, de Jong J, van der Poel HG, et al. Histopathological Features of MRI-Invisible Regions of Prostate Cancer Lesions. J Magn Reson Imaging 2019. doi:10.1002/jmri.26933.

[24] Aluwini S, van Rooij P, Hoogeman M, Kirkels W, Kolkman-Deurloo I-K, Bangma C. Stereotactic body radiotherapy with a focal boost to the MRI-visible tumor as monotherapy for low- and intermediate-risk prostate cancer: early results. Radiat Oncol 2013;8:84. doi:10.1186/1748717X-8-84.

[25] Kotecha R, Djemil T, Tendulkar RD, Reddy CA, Thousand RA, Vassil A, et al. Dose-Escalated Stereotactic Body Radiation Therapy for Patients With Intermediate- and High-Risk Prostate Cancer: Initial Dosimetry Analysis and Patient Outcomes. Int J Radiat Oncol 2016;95:960-4. doi:10.1016/J.IJROBP.2016.02.009. 
[26] Herrera FG, Valerio M, Berthold D, Tawadros T, Meuwly J-Y, Vallet V, et al. 50 Gy Stereotactic Body Radiation Therapy to the Dominant Intra-Prostatic Nodule: Results from a Phase la/b Trial. Int J Radiat Oncol Biol Phys 2018. doi:10.1016/j.ijrobp.2018.09.023.

[27] McDonald AM, Dobelbower MC, Yang ES, Clark GM, Jacob R, Kim RY, et al. Prostate Stereotactic Body Radiation Therapy With a Focal Simultaneous Integrated Boost: Acute Toxicity and Dosimetry Results From a Prospective Trial. Adv Radiat Oncol 2019;4:90-5. doi:10.1016/J.ADRO.2018.09.007.

[28] Weinreb JC, Barentsz JO, Choyke PL, Cornud F, Haider MA, Macura KJ, et al. PI-RADS Prostate Imaging - Reporting and Data System: 2015, Version 2. Eur Urol 2016;69:16-40. doi:10.1016/j.eururo.2015.08.052.

[29] Salembier C, Villeirs G, De Bari B, Hoskin P, Pieters BR, Van Vulpen M, et al. ESTRO ACROP consensus guideline on $\mathrm{CT}$ - and $\mathrm{MRI}$-based target volume delineation for primary radiation therapy of localized prostate cancer. Radiother Oncol 2018;127:49-61. doi:10.1016/j.radonc.2018.01.014.

[30] Gladwish A, Pang G, Cheung P, D'Alimonte L, Deabreu A, Loblaw A. Prostatic displacement during extreme hypofractionated radiotherapy using volumetric modulated arc therapy (VMAT). Radiat Oncol 2014;9:262. doi:10.1186/s13014-014-0262-y.

[31] Gay HA, Barthold HJ, O'Meara E, Bosch WR, El Naqa I, Al-Lozi R, et al. Pelvic normal tissue contouring guidelines for radiation therapy: a Radiation Therapy Oncology Group consensus panel atlas. Int J Radiat Oncol Biol Phys 2012;83:e353-62. doi:10.1016/j.ijrobp.2012.01.023.

[32] Draulans C, De Roover R, van der Heide UA, Haustermans K, Pos F, Smeenk RJ, et al. Stereotactic body radiation therapy with optional focal lesion ablative microboost in prostate cancer: Topical review and multicenter consensus. Radiother Oncol 2019;140:131-42. doi:10.1016/j.radonc.2019.06.023.

[33] Dörr W. Radiobiology of tissue reactions. Ann ICRP 2015;44:58-68. doi:10.1177/0146645314560686.

[34] Lips IM, Dehnad H, van Gils CH, Boeken Kruger AE, van der Heide UA, van Vulpen M. Highdose intensity-modulated radiotherapy for prostate cancer using daily fiducial marker-based position verification: acute and late toxicity in 331 patients. Radiat Oncol 2008;3:15. doi:10.1186/1748-717X-3-15.

[35] Mottet N, Bellmunt J, Bolla M, Briers E, Cumberbatch MG, De Santis M, et al. EAU-ESTRO-SIOG Guidelines on Prostate Cancer. Part 1: Screening, Diagnosis, and Local Treatment with Curative Intent. Eur Urol 2016;71:618-29. doi:10.1016/j.eururo.2016.08.003.

[36] Van As NJ, Brand D, Tree A, Ostler PJ, Chu W, Loblaw A, et al. PACE: Analysis of acute toxicity in PACE-B, an international phase III randomized controlled trial comparing stereotactic body radiotherapy (SBRT) to conventionally fractionated or moderately hypofractionated external beam radiotherapy (CFMHRT) for locali. J Clin Oncol 2019;37:1-1. doi:10.1200/jco.2019.37.7_suppl.1.

[37] Chinnachamy AN, Chopra S, Krishnatry R, Kannan S, Thomas B, Mahantshetty U, et al. Evaluation of Interobserver and Interscale Agreement in Assessing Late Bowel Toxicity after Pelvic Radiation in Patients with Carcinoma of the Cervix. Jpn J Clin Oncol 2013;43:508-14. doi:10.1093/jjco/hyt028. 